\title{
Impact Evaluation of Assimilating Surface Sensitive Infrared Radiance Observations over Land and Sea Ice from Observing System Simulation Experiments
}

\author{
S. K. Dutta, L. Garand, and S. Heilliette \\ Meteorological Research Division, Data Assimilation and Satellite Meteorology Section, Environment Canada, \\ Dorval, QC, Canada H9P 1 J3 \\ Correspondence should be addressed to L. Garand; louis.garand@ec.gc.ca
}

Received 5 February 2015; Accepted 14 May 2015

Academic Editor: Tomoo Ushio

Copyright (C) 2015 S. K. Dutta et al. This is an open access article distributed under the Creative Commons Attribution License, which permits unrestricted use, distribution, and reproduction in any medium, provided the original work is properly cited.

In this study, Observing System Simulation Experiments (OSSEs) are conducted to analyze the impact of assimilating surface sensitive infrared radiance observations over land and sea ice. This type of assimilation has not yet been successfully implemented at operational weather centers. Infrared radiance from AIRS (Atmospheric Infrared Sounder) and IASI (Infrared Atmospheric Sounding Interferometer) is simulated from the Nature Run (NR) provided by European Centre for Medium-Range Weather Forecasts and assimilated in a 3D-Var. analysis system. A control simulation was generated excluding the new data source, but including all data assimilated operationally at the Canadian Meteorological Center. Experiments were conducted allowing surface sensitive channels to be assimilated over all surfaces or excluding Polar Regions. Resulting forecasts were intercompared and validated against NR fields. Results indicate significant positive impacts in the tropics and Southern Hemisphere extratropics and more modest impacts in the Northern Hemisphere extratropics. Some limitations of the OSSE approach are identified, linked to the different forecast systems used for the NR and the assimilation and higher cloud contamination in Polar Regions. This analysis provides useful insight in preparation for the assimilation of real radiance observations.

\section{Introduction}

Observing System Simulation Experiments (OSSEs) are an effective means for evaluating the potential impact of proposed observing systems on numerical weather prediction (NWP) (Arnold Jr. and Dey [1]; Hoffman et al. [2]; Atlas [3]; Atlas et al. [4]). In remote sensing in general, the evaluation of an observing system from simulated data is very useful since the investigator benefits from a predefined "truth," allowing sensitivity studies to key parameters on the quality of the retrievals. Here we make use of a comprehensive weather analysis system involving simulation of all data types currently used in operations at the Canadian Meteorological Center (CMC) (Charron et al. [5]) as well as simulation of the new data type under investigation. For this type of OSSE, a free model run defines the "true" atmospheric state. This forecast, referred to as "Nature Run" (NR) is used to generate the synthetic observations needed for the experimentation.
The impact of assimilating the simulated observations is then assessed by comparing resulting forecasts with NR analyses, with and without assimilation of the new data type. This process also helps evaluating the data assimilation methodology and the sensitivity to quality control criteria.

OSSEs involving satellite observations date back to 1970s, when many experiments were conducted (Gordon et al. [6]; Kasahara and Williamson [7]) showing the possibility of substantial improvement in NWP with the assimilation of satellite derived temperature and wind observations. More recently, the beneficial impact from the SWIFT stratospheric wind and ozone observations was assessed from an OSSE (Lahoz et al. [8]). Among recent studies, Otkin [9] has conducted an OSSE using an ensemble Kalman Filter at convection scale. He examined the impact on the accuracy of atmospheric analyses of assimilating window infrared brightness temperatures in both clear and cloudy sky conditions. At the Joint Center for Satellite Data Assimilation in United 
States, a Joint OSSE Project was initiated (Zhu et al. [10]) with goal to provide simulated data for various studies. Synthetic satellite observations were simulated from NR from the Joint OSSE Project using Community Radiance Transfer Model, followed by assimilation impact evaluation in the Gridpoint Statistical Interpolation system. Kleist and Ide [11] carried out an OSSE to evaluate the performance of their hybrid variational-ensemble data assimilation system at the National Centre for Environmental Prediction (NCEP).

Environment Canada (Garand et al. [12]) evaluated from OSSEs the benefits of assimilating atmospheric motion vectors (AMVs) derived from highly elliptical orbit imagery covering the Polar Regions. Wang et al. [13] designed an OSSE for assessing the positive impact of the future infrared sounder onboard Meteosat Third Generation geostationary meteorological satellite system. Heilliette et al. [14] described the procedures involved in the simulation of infrared radiance and their quality control for an OSSE. They evaluated the level of cloud contamination potentially affecting real radiance observations as a function of wavelength. This OSSE study allowed them to evaluate the residual biases linked to cloud contamination. The consistency of the impact of assimilating of real and simulated AIRS and IASI radiance data demonstrated the realism of OSSE.

The purpose of this work is to study the potential impact of assimilating surface sensitive infrared radiance observations over land and sea ice. Currently, this is not achieved yet at operational NWP centers, which represents an underuse of that rich information on low level temperature and humidity. Over land, only radiance which is not sensitive to the surface or to low clouds is assimilated. The challenge is to demonstrate the added value on top of that provided by other data types such as surface, aircraft, ground profiler observations, and satellite observations. The strategy is to work first with simulated data to get a sense of the limitations before actually attempting this type of assimilation in a real system. The additional data come from hyperspectral infrared sounders AIRS (Atmospheric Infrared Sounder) and IASI (Infrared Atmospheric Sounding Interferometer). Section 2 describes the experimental setup and the assimilation approach. Section 3 provides results based on twomonth assimilation cycles. Section 4 concludes the paper, evaluating best options for the next phase consisting of using real observations.

\section{Experimental Setup}

2.1. Nature Run and Simulated Observations. The present study is based on the OSSE configuration used in previous studies $[12,14]$. The Nature Run used for the experiment is obtained from the European Centre for Medium-Range Weather Forecasts (ECMWF) covering the period from 10 May 2005 to 31 May 2006; see Reale et al. [15] and Masutani et al. $[16,17]$. The horizontal resolution of the NR fields produced at 3-hour interval is about $40 \mathrm{~km}$. It has 91 vertical levels with top at $0.02 \mathrm{hPa}$. The sea surface temperature and ice cover used for the generation of NR were provided by NCEP. Before extraction of the simulated data from the NR fields, it was first interpolated onto the CMC's global forecast model grid which has a similar resolution, that is, $\sim 33 \mathrm{~km}$ and 80 levels, with top at $0.1 \mathrm{hPa}$. All data assimilated operationally in 2008-2009 were simulated (plus additional AIRS and IASI channels), amounting to about $6 \mathrm{M}$ observations. A 3D-Var. assimilation methodology is used for the assimilation of observations in a $6 \mathrm{~h}$ cycle (Barker et al. [18]). Forecasts up to 5 days were launched every $12 \mathrm{~h}$ using the operational GEM (Global Environmental Multiscale) model [5]. These forecasts were validated against the NR interpolated, as mentioned, at the resolution of GEM. Cycles were run for two months covering the period from 15 December 2005 to 27 February 2006. The sea surface temperature and the albedo fields used are those used at the CMC at that time and thus differ from those of the NR. Since data types such as IASI and recent microwave sensors were not available at the time of the NR, the positions of all simulated observations were those available for the exact same period, but three years later, that is, 2008-2009. Rochon et al. [19] described the general procedure for the perturbation of the observations consistent with their associated errors. The appropriate level of perturbations for AIRS and IASI simulated observations was further evaluated [14]. These earlier studies used 87 AIRS channels and 62 IASI channels. Here, following the current operational practice at the CMC, we consider 142 channels for each sensor. Figure 1 shows the assigned observation error for the 142 IASI channels, identifying specifically surface sensitive ones. These errors are largely above radiometric noise to compensate for other factors such as spatiotemporal error correlations (Garand et al. [20]). The surface sensitive channels are located in the region $8.0-13.8 \mu \mathrm{m}$ and at wavelengths lower than $5.3 \mu \mathrm{m}$. The corresponding figure for AIRS (not shown) is very similar. The spectral surface emissivity database is that provided by CERES (Clouds and the Earth's Radiant Energy System) (Garand [21]).

2.2. Experiments. A set of three simulations are performed: the control simulation "CNTLEXP0" and two experimental simulations "OSSEXP1" and "OSSEXP2" (labels appearing in graphs), hereafter referred to as CNTL, EXP1, and EXP2, respectively. As detailed in our previous OSSE work [12], assimilated observations include those from wind profilers, radiosondes, land stations, scatterometers, ships, buoys, atmospheric motion vectors, aircrafts, GPS (Ground Positioning System) radio occultation, microwave radiance from several sensors, and infrared radiance from AIRS, IASI, and geostationary satellites.

In addition to these observations, infrared radiance from surface sensitive channels of AIRS and IASI has been assimilated over land and sea ice under restrictive conditions. High resolution topography data is used as one of the limiting criteria. A local standard deviation of $50 \mathrm{~m}$ in the height of topography (at $50 \mathrm{~km}$ scale) is imposed in order to limit the assimilation to relatively flat, uniform terrain. That criterion eliminates about $35 \%$ of land masses. Furthermore, the additional AIRS and IASI radiance observations are assimilated in regions characterized by high surface emissivity, that is, $>0.97$, for surface sensitive channels in order to limit errors due to that important parameter. Over sea ice, a complete ice cover is required (ice fraction $>0.99$ ). Over land, a local estimate of 


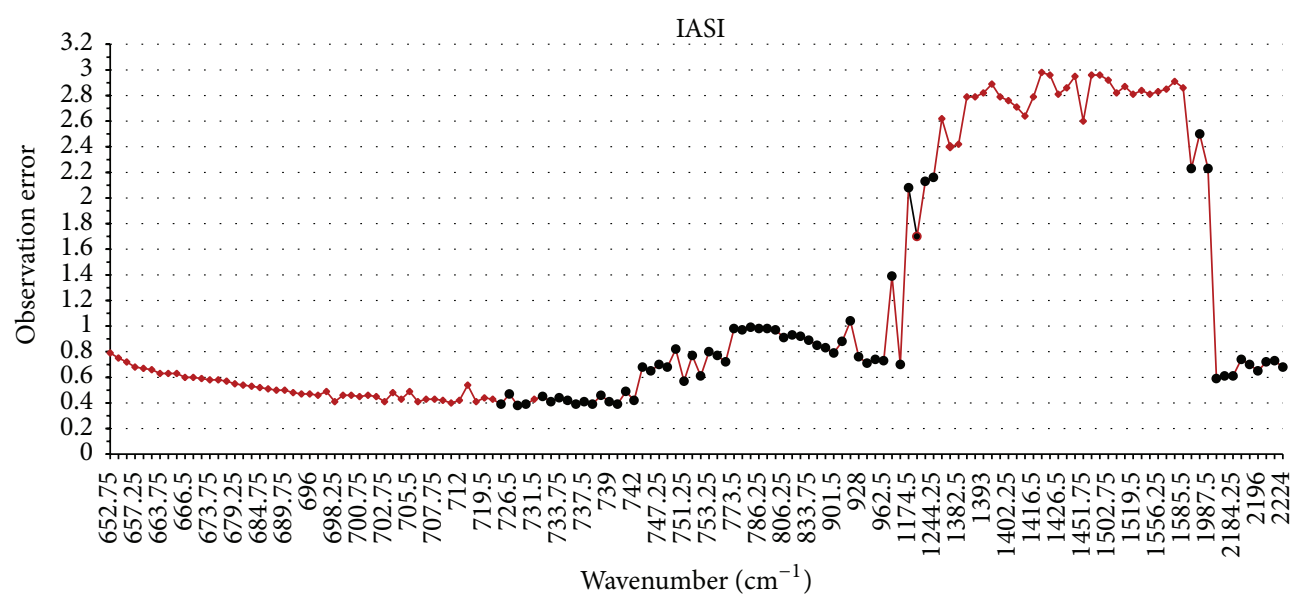

FIGURE 1: Assigned observation error as a function of wavenumber $\left(\mathrm{cm}^{-1}\right)$ for the 142 IASI channels assimilated. Black dots identify surface sensitive channels.

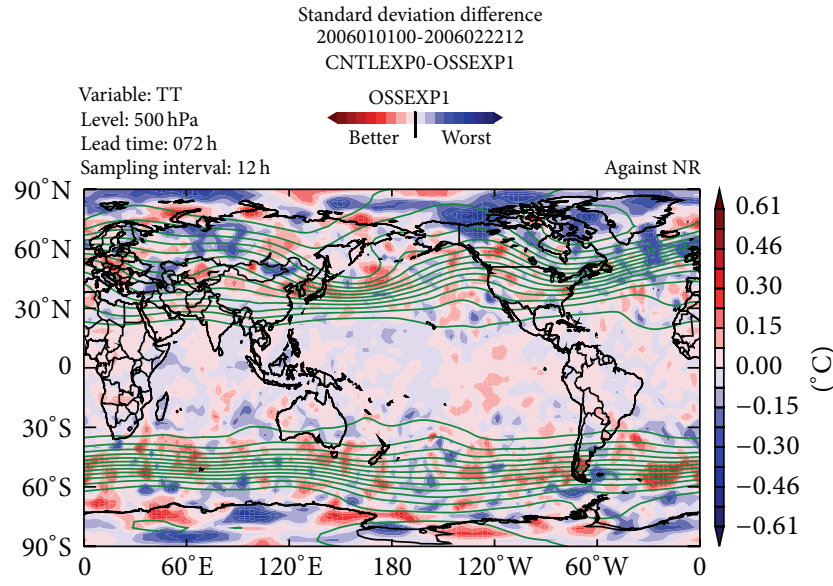

(a)

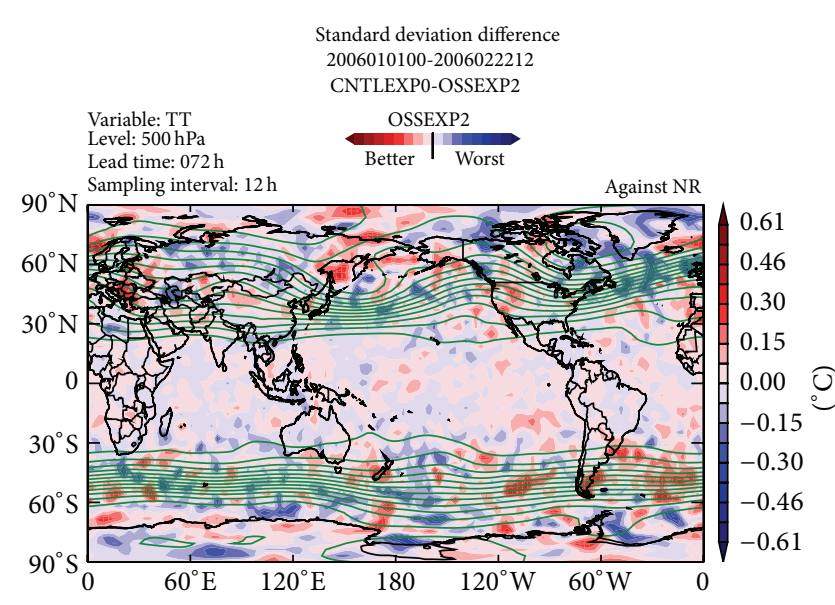

(b)

Figure 2: Global distribution of std. difference of $72 \mathrm{~h} 500 \mathrm{hPa}$ temperature between CNTL and (a) EXP1 and (b) EXP2 evaluated against the Nature Run. Blue regions indicate deterioration while red regions are indicative of improvement with respect to the control.

surface skin temperature is obtained using a window channel in regions assumed clear. That estimate must be within $4 \mathrm{~K}$ of that from the model first guess for assimilation to be allowed. Simulated radiance is assumed to be unbiased since it was computed from the same radiative transfer model (RTM) as the one used in the assimilation. This choice may contribute to an overestimation of positive impact, but we recall that a different forecast model and a different SST are used in the NR and assimilation to avoid the situation of "identical twin" experiments (Cardinali et al. [22]; Masutani et al. [23]). The criterion differentiating EXP1 from EXP2 is based on surface coverage: the latter excludes latitudinal regions poleward of $60^{\circ} \mathrm{N} / \mathrm{S}$, that is, in both hemispheres. Limitations and quality control criteria defined above are a result of a number of previous sensitivity tests. Thus, even with simulated data, a prudent approach is taken to define best conditions for a successful assimilation.

\section{Results}

3.1. A Global View. The first two-month experiment, EXP1, included the assimilation of surface sensitive infrared radiance observations over all surfaces where no clouds are detected. This is the only difference with respect to the control experiment in which these observations are assimilated over ice-free oceans only. Standard deviation (std.) differences between CNTL and EXP1 forecasts, computed against the ECMWF Nature Run, were computed. Figure 2(a) depicts the $72 \mathrm{~h}$ forecast $500 \mathrm{hPa}$ std. difference over the global domain. Figure 2(b) shows corresponding differences between the control and EXP2. Blue regions indicate deterioration while red regions are indicative of improvement with respect to the control. For EXP1, negative impacts are noted mostly over the North Polar Region $\left(60^{\circ}-90^{\circ} \mathrm{N}\right)$, but also in the Antarctica Region $\left(60^{\circ}-90^{\circ} \mathrm{S}\right)$, which motivated 


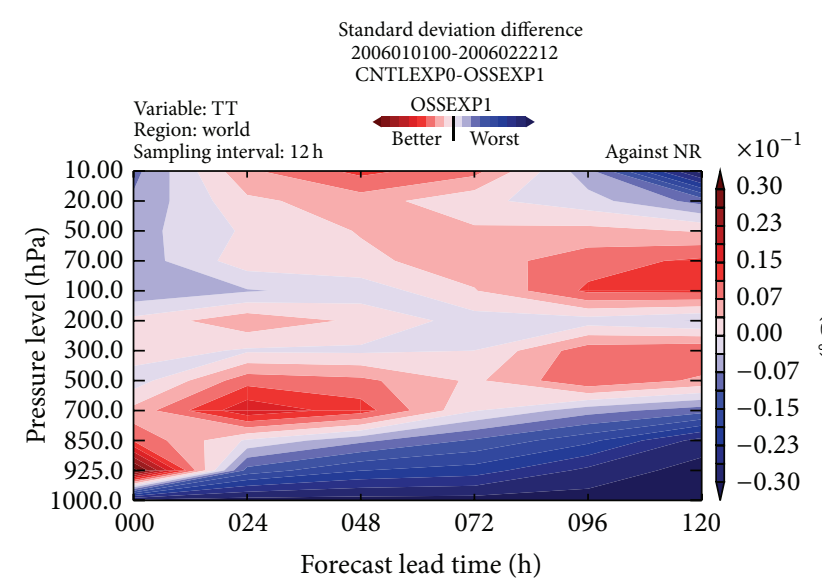

(a)

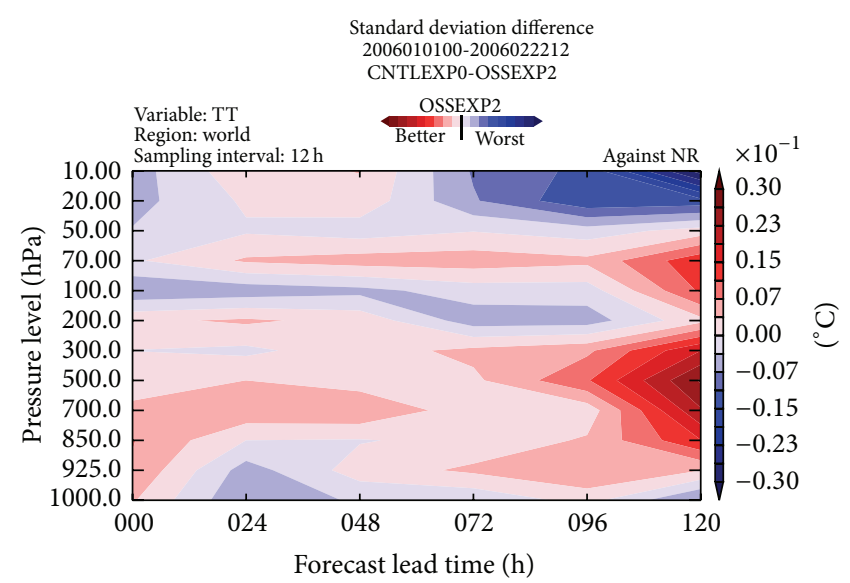

(b)

FIGURE 3: Profile of std. difference between CNTL and (a) EXP1 and (b) EXP2 temperature, computed against the Nature Run over the global domain, as a function of lead time into the forecast. Blue regions indicate deterioration while red regions are indicative of improvement with respect to the control.

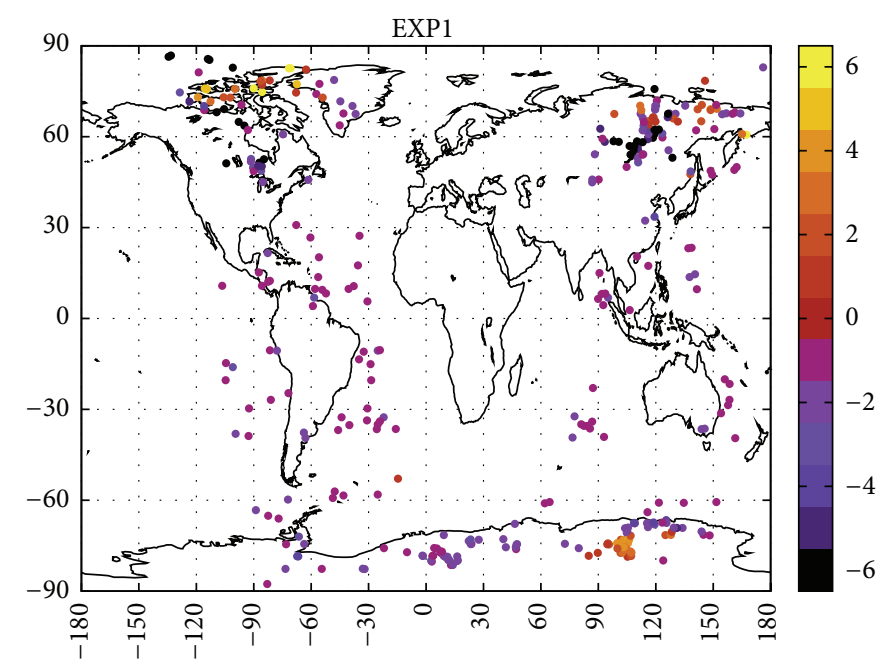

(a)

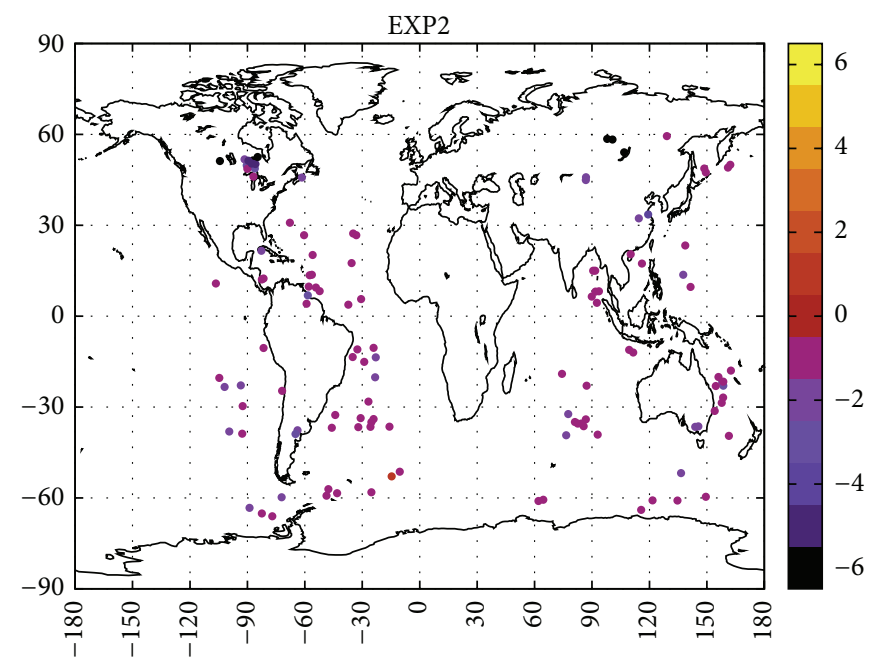

(b)

FIGURE 4: Difference between all-sky and clear sky brightness temperature (K) assimilated in experiments EXP1 (a) and EXP2 (b) for AIRS channel 787 for a $6 \mathrm{~h}$ period centered on 15 February 2006, 18 UTC.

the latitudinal restrictions imposed in EXP2. Much improved results are noted in Figure 2(b). Figures 3(a) and 3(b) display the corresponding vertical profile of std. differences of temperature between each experiment and the control, as a function of forecast lead time over the global domain. A negative impact is noted at low level for EXP1. The depth of this negative impact increases gradually with time into the forecast from the surface to $700 \mathrm{hPa}$. In contrast, results are again much more convincing in the case of EXP2, with positive impacts noted over most of the troposphere.

The question arises: why the assimilation appears to be more problematic in Polar Regions? A recognized difficulty is that of correct identification of cloud conditions. Frequent surface inversions with often nearly isothermal profiles above the boundary layer make both clear sky identification and subsequent assimilation a challenge. Here, as discussed elsewhere [14], radiance observations were simulated for all-sky conditions followed by cloud parameter detection as done with real data. Thus, some cloud contamination is allowed to represent conditions prevailing in real assimilation. To investigate that issue, the differences between clear sky and all-sky brightness temperatures for AIRS window channel 787 $(10.9 \mu \mathrm{m})$ were computed for several simulated orbits. The result is shown in Figures 4(a) and 4(b) for pixels selected for assimilation as part of EXP1 and EXP2, respectively, for a specific orbit. It is seen that cloud contamination is generally modest in EXP2, most often with negative differences between all-sky and clear radiance, and rarely exceeding $2 \mathrm{~K}$. In contrast in EXP1, cloud contamination is often in excess of $4 \mathrm{~K}$ and can be of both signs. Positive differences 


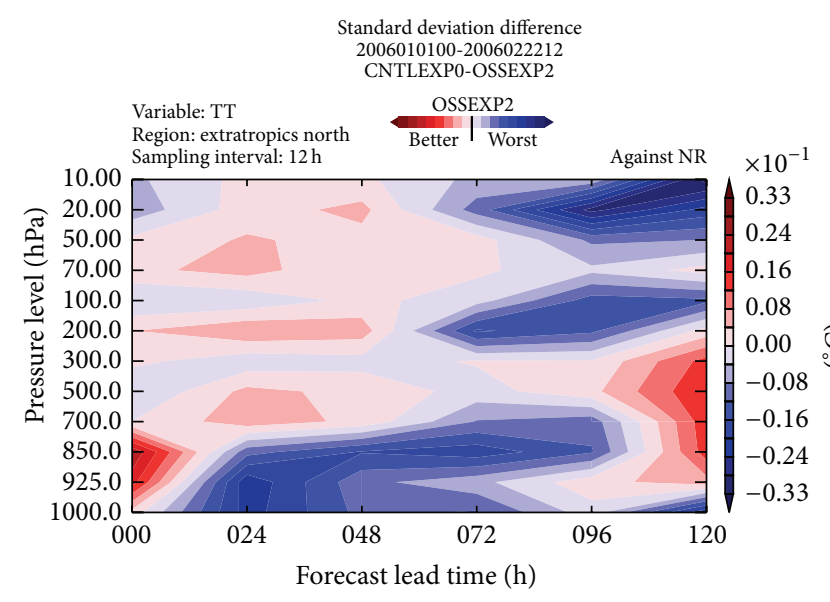

(a)

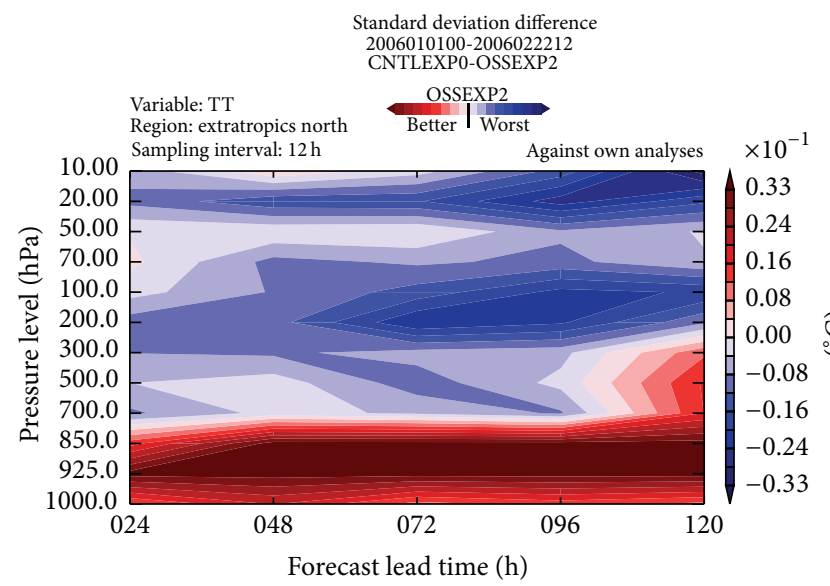

(c)

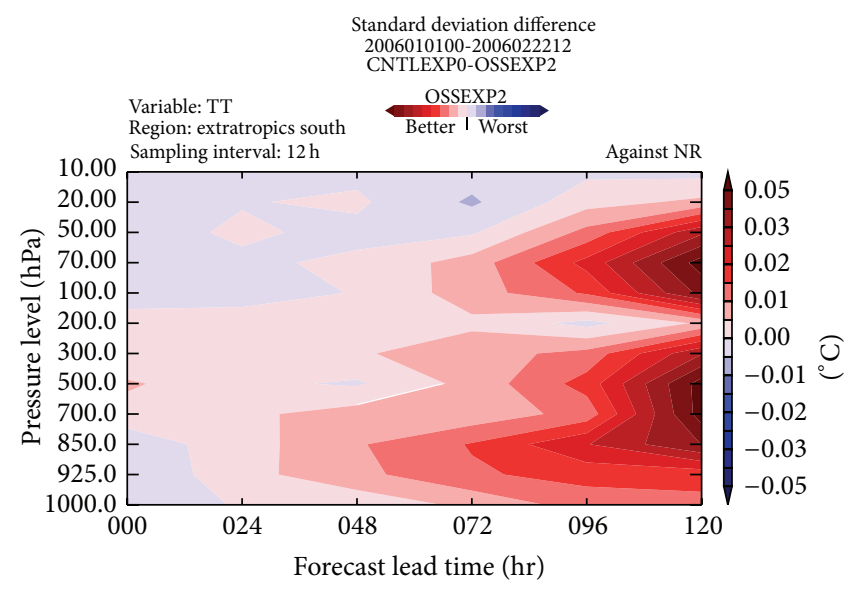

(b)

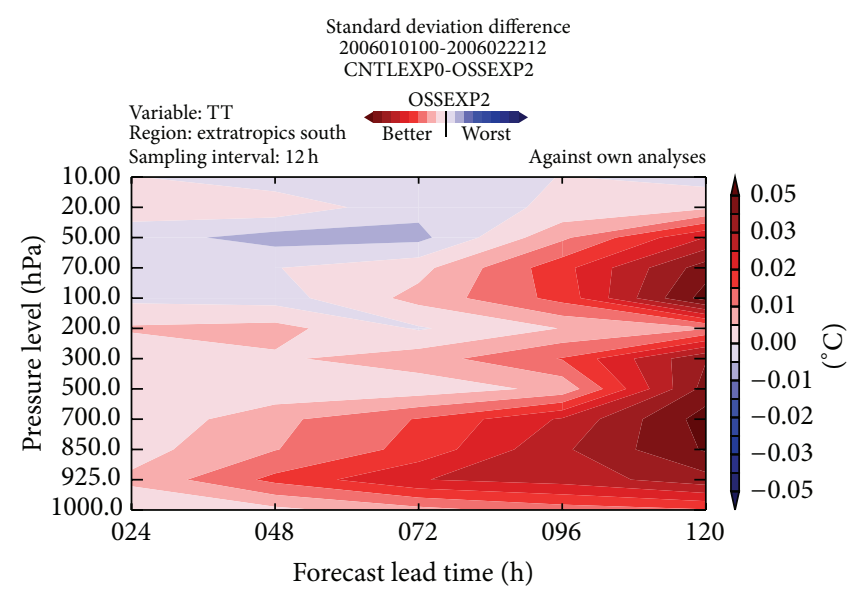

(d)

Figure 5: Standard deviation difference between EXP2 temperature $\left({ }^{\circ} \mathrm{C}\right)$ forecasts and Nature Run $(\mathrm{a}, \mathrm{b})$ and own cycle $(\mathrm{c}, \mathrm{d})$ analyses for the Northern Hemisphere $(a, c)$ and Southern Hemisphere $(b, d)$ extratropics regions. Blue regions indicate deterioration while red regions are indicative of improvement with respect to the control.

can occur in surface inversion situations with a relatively warm cloud present at the top of the inversion. Radiance observations are accepted for assimilation on the basis of estimated clear sky condition combined with thresholds on differences between the simulated observations and that computed from the model first guess (a short forecast, typically $6 \mathrm{~h}$ ). Quality control may fail in situations of errors of similar magnitude and the same sign for the model equivalent of the observation and the observation wrongly perceived as clear. An additional difficulty linked to the OSSE setup is that the NR is based on the ECMWF model while the assimilation uses a different model, as recommended to avoid overoptimistic impact inferences (Stoffelen et al. [24]). The physics affecting surface and boundary layer processes are quite different as well as related error characteristics. These differences may impact more significantly the radiance quality control and the assimilation process in Polar Regions as suggested from Figure 4 results. Similar issues, with more modest effects, were raised in previous work [15] linked to the different sea surface temperature analysis used in the NR and in the OSSE. Further insight can be gained from the comparison of forecasts evaluated against the NR and against the analysis generated in the cycle, referred to as "OWN" analysis. Figure 5 depicts this comparison in the same manner as in Figure 3 for the Northern and Southern extratropics (latitudes $20^{\circ}-70^{\circ} \mathrm{N} / \mathrm{S}$ ). It is apparent that there is a high degree of consistency in the Southern extratropics results between forecasts evaluated against OWN and NR analyses, both indicating a clear positive impact over the entire troposphere. This is also noted in the tropics region (not shown). However, Northern Hemisphere OWN and NR results are in opposition, strongly positive at low levels (700 hPa to surface) for the validation against OWN analyses and rather negative for the validation versus the NR (for $24 \mathrm{~h}$ to $96 \mathrm{~h}$ forecasts). Better agreement is seen at $120 \mathrm{~h}$ above $850 \mathrm{hPa}$. It appears that, in cold atmospheres, the assimilation is not successful in reproducing well the NR atmosphere. Consequently, impact results for that region do not appear as conclusive as those for regions more to the south.

3.2. EXP2 Detailed Impacts. In EXP2, the volume of assimilated radiance observations for surface sensitive channels 

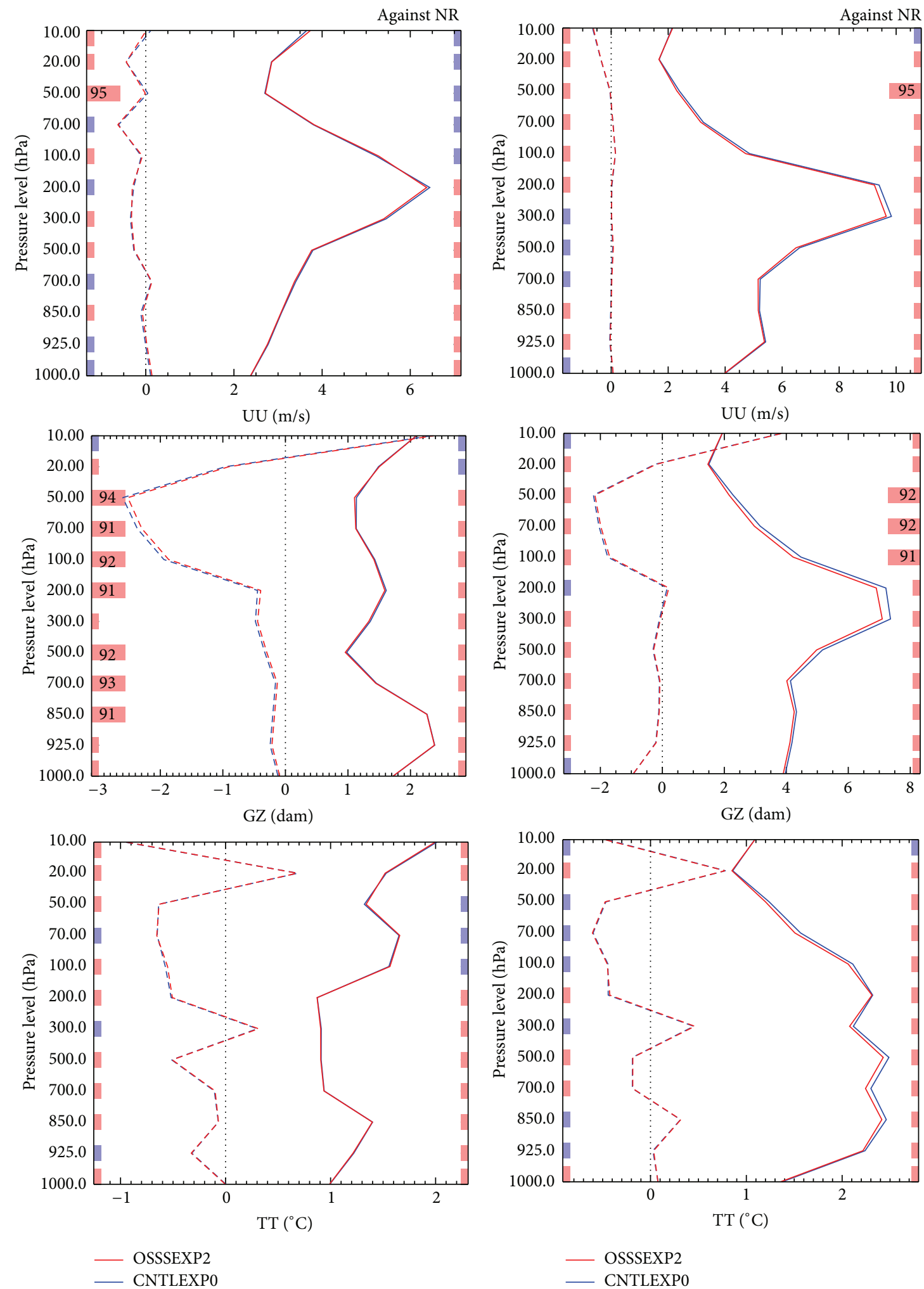

(a)

(b)

FIgure 6: Profile of standard deviation differences (solid lines) for EXP2 (red) and CNTL (blue) against the NR for the tropics (a) and extratropics south (b) regions at $120 \mathrm{hr}$; broken lines represent the bias. The level of significance above $90 \%$ is indicated. The three variables $\mathrm{UU}$ (zonal wind, $\mathrm{m} / \mathrm{s}$ ), GZ (geopotential, dam), and TT (temperature, ${ }^{\circ} \mathrm{C}$ ) are identified on the $x$ axis. Red markers on each side denote superiority of EXP2 and the blue markers denote superiority of CNTL. 

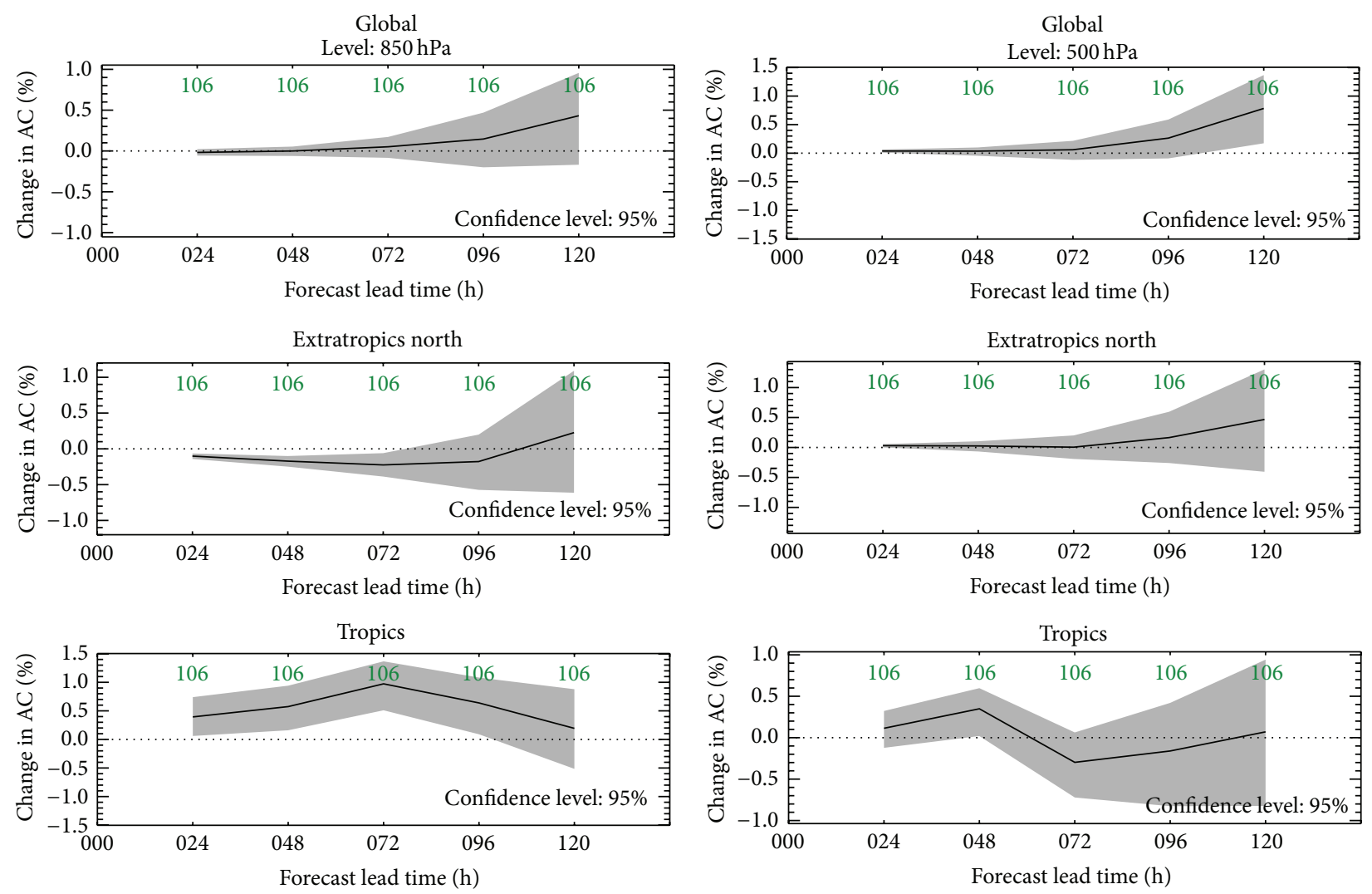

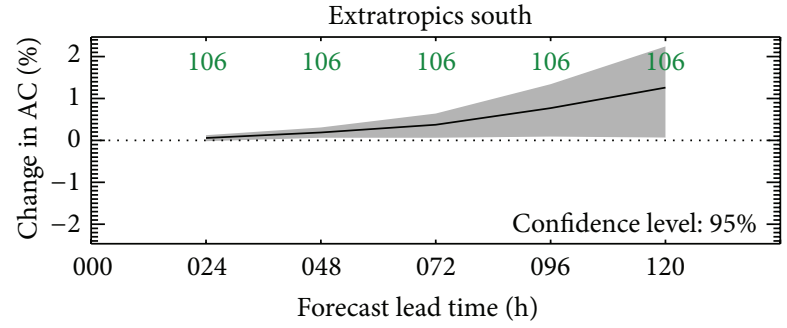

(a)

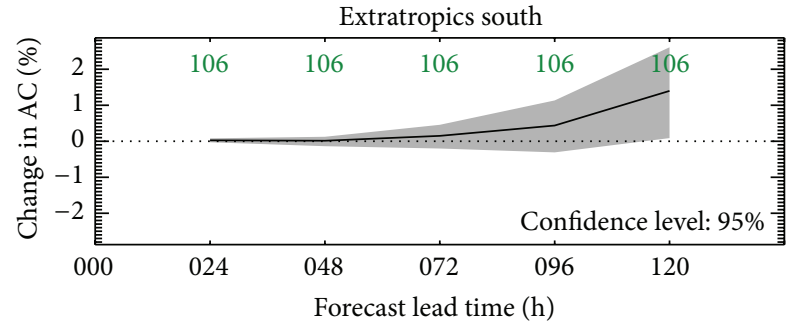

(b)

FIGURE 7: Differences in anomaly correlation (\%) at $850 \mathrm{hPa}$ (a) and $500 \mathrm{hPa}$ (b) for temperature between EXP2 and CNTL, validated against the NR, as a function of forecast lead time for the four regions indicated. Gray shading above the dotted line indicates that the impact is positive at 95\% significance level.

increases by about $15 \%$ with respect to CNTL. Both AIRS and IASI sensors provide additional observations over land at about 800 locations per day. Here we examine various common measures of impact pertaining to EXP2. Figure 6 depicts the vertical profile of standard deviation differences (solid lines) for EXP2 and CNTL evaluated against the NR for $120 \mathrm{~h}$ forecasts pertaining to the tropics and extratropics south regions. Broken lines represent the corresponding bias. Parameters are designated as UU (Zonal Wind), GZ (Geopotential), and TT (temperature). A positive impact is noted for the three variables from $200 \mathrm{hPa}$ to the surface. The positive impact is most pronounced in the extratropics south region. Results for the extratropics north region (not shown) are qualitatively similar to those seen in the tropics.
A common measure used in NWP to evaluate forecasts is anomaly correlation. Figure 7 presents $850 \mathrm{hPa}$ and $500 \mathrm{hPa}$ temperature anomaly correlation differences between EXP2 and CNTL (evaluated against the NR) as a function of lead time for the globe and the three main latitudinal regions based on a sample of 106 forecasts. Gray shading above the zero line indicates that the difference is positively significant at $95 \%$ confidence level. The impact is significant in the tropics at $850 \mathrm{hPa}$ while it is neutral at $500 \mathrm{hPa}$. Results are significantly positive globally at day 5 at $500 \mathrm{hPa}$ and at both levels in the Southern Hemisphere. The impact occurs at shorter lead times in the tropics.

Results derived from time series provide additional insight, depicting the level of consistency in scores from 


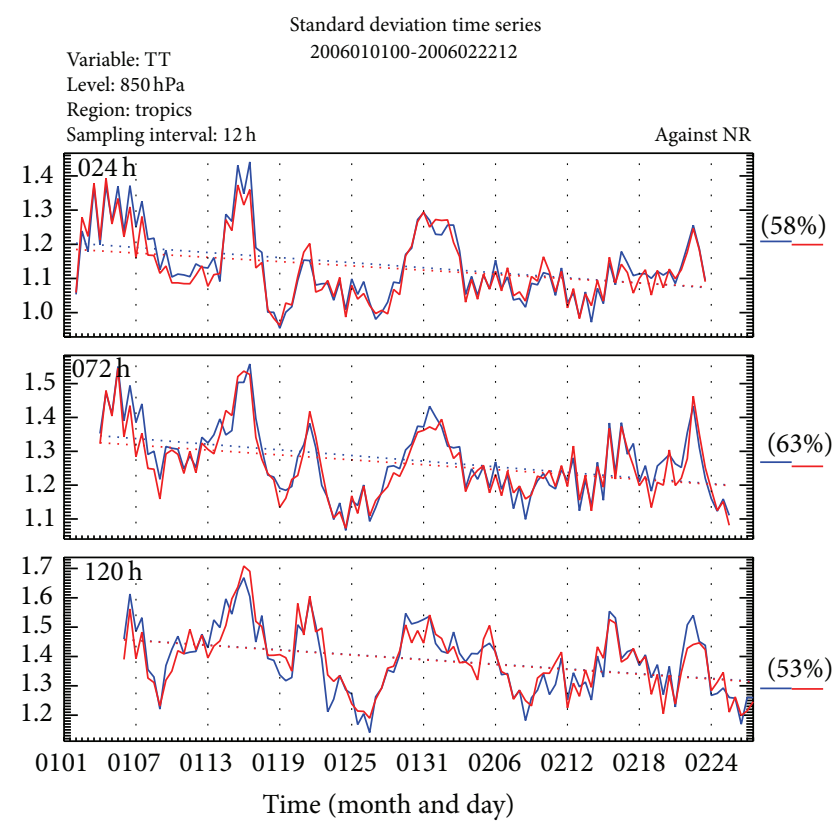

- OSSEXP2
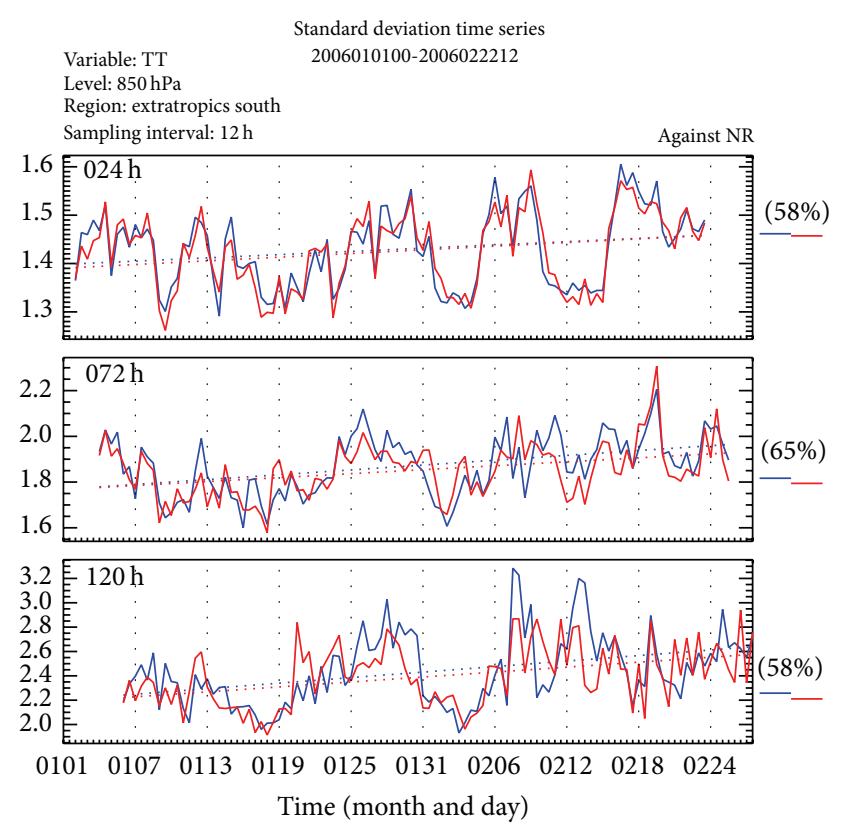

OSSEXP2
CNTLEXP0

(a)

(b)

FIgURE 8: Standard deviation time series against the NR of EXP2 (red) and CNTL (blue) $850 \mathrm{hPa}$ temperature (K) for $24 \mathrm{~h}, 72 \mathrm{~h}$, and $120 \mathrm{~h}$ forecasts for the tropics (a) and extratropics south (b) regions. Indicated on the right is the percentage of cases where EXP2 improves over CNTL as well as the global std. difference.

individual forecasts. Figure 8 presents a comparison between EXP2 and CNTL time series of $850 \mathrm{hPa}$ standard deviation of temperature against the NR for the tropics (Figure 8(a)) and extratropics south (Figure $8(\mathrm{~b})$ ) regions. The series is shown for $24 \mathrm{~h}, 72 \mathrm{~h}$, and $120 \mathrm{~h}$ forecasts. The percentage of the time EXP2 is superior to CNTL which is shown on the right. That percentage is in the range $53-65 \%$ with the largest differences in std. noted for $72 \mathrm{~h}$ and $120 \mathrm{~h}$ forecasts in the extratropics south region. Such systematic positive impacts, including large improvements on individual cases, are worth emphasizing. Thus all results presented in this section are indicative of significant positive impacts in the tropics and southern extratropics.

\section{Conclusion}

In this study, Observing System Simulation Experiments were conducted to evaluate the potential impact of assimilating infrared radiance observations over land and sea ice. OSSEs offer the advantage of benefiting from a predefined "true" atmospheric state allowing sensitivity studies in a controlled environment. As shown here, OSSEs provide much insight on strengths and limitations to expect with such an assimilation using real observations. It was found, in particular, that there are inherent difficulties in Polar Regions, with negative consequences spreading to lower latitudes. These are likely linked to the assimilation of cloud contaminated radiance. However, it was also shown that, for this specific OSSE, cloud contamination may be enhanced due to the difference between the atmospheric models used in the NR and in the assimilation. A lack of consistency was noted between results evaluated against own and Nature Run analyses for Northern Hemisphere extratropics region. This inconsistency is likely linked to differences in boundary layer physics of the ECMWF and EC models. In contrast, consistent and largely positive impact results were obtained for the tropics and Southern Hemisphere regions. Since Environment Canada is planning to upgrade the resolution of its global model to $15 \mathrm{~km}$, future OSSE work will require higher resolution Nature Run. This is now possible using the recently released two-year Nature Run at $7 \mathrm{~km}$ resolution and $30 \mathrm{~min}$ intervals by the Global Modeling and Assimilation Office (http://gmao.gsfc.nasa.gov/global_mesoscale/G5NR/).

Assimilation with real data is a natural continuation to this study. The context is now favorable, with Environment Canada now benefiting from a higher resolution analysis and the implementation of a $4 \mathrm{D}$ ensemble variational assimilation system (Buehner et al. [25]), with flow dependent background errors from an Ensemble Kalman Filter system. Data from a second IASI instrument as well as from CrIS (Crosstrack Infrared Sounder, onboard Suomi satellite) are now available for a total of four infrared hyperspectral sounders. Experiments are now in progress, inspired by the prudent approach used in this study.

\section{Conflict of Interests}

The authors declare that there is no conflict of interests regarding the publication of this paper. 


\section{References}

[1] C. P. Arnold Jr. and C. H. Dey, "Observing-systems simulation experiments: past, present and future," Bulletin of American Meteorological Society, vol. 67, no. 6, pp. 687-695, 1986.

[2] R. N. Hoffman, C. Grassotti, R. G. Isaacs, J.-F. Louis, T. Nehrkorn, and D. C. Norquist, "Assessment of the impact of simulated satellite lidar wind and retrieved $183 \mathrm{GHz}$ water vapor observations on a global data assimilation system," Monthly Weather Review, vol. 118, no. 12, pp. 2513-2542, 1990.

[3] R. Atlas, "Atmospheric observations and experiments to assess their usefulness in data assimilation," Journal of the Meteorological Society of Japan, vol. 75, no. 1 B, pp. 111-130, 1997.

[4] R. Atlas, R. N. Hoffman, S. M. Leidner et al., "The effects of marine winds from scatterometer data on weather analysis and forecasting," Bulletin of the American Meteorological Society, vol. 82, no. 9, pp. 1965-1990, 2001.

[5] M. Charron, S. Polavarapu, M. Buehner et al., "The stratospheric extension of the Canadian global deterministic medium-range weather forecasting system and its impact on tropospheric forecasts," Monthly Weather Review, vol. 140, no. 6, pp. 19241944, 2012.

[6] C. T. Gordon, L. Umscheid Jr., and K. Miyakoda, "Simulation experiments for determining wind data requirements in the tropics," Journal of the Atmospheric Sciences, vol. 29, no. 6, pp. 1064-1075, 1972.

[7] A. Kasahara and D. Williamson, "Evaluation of tropical wind and reference pressure measurements: numerical experiments for observing systems," Tellus, vol. 24, no. 2, pp. 100-115, 1972.

[8] W. A. Lahoz, R. Brugge, D. R. Jackson et al., "An observing system simulation experiment to evaluate the scientific merit of wind and ozone measurements from the future SWIFT instrument," Quarterly Journal of the Royal Meteorological Society, vol. 131, no. 606, pp. 503-523, 2005.

[9] J. A. Otkin, "Clear and cloudy sky infrared brightness temperature assimilation using an ensemble Kalman filter," Journal of Geophysical Research D: Atmospheres, vol. 115, no. 19, Article ID D19207, 2010.

[10] T. Zhu, F. Weng, M. Masutani, and J. S. Woollen, "Synthetic radiance simulation and evaluation for a Joint Observing System Simulation Experiment," Journal of Geophysical Research D: Atmospheres, vol. 117, no. 23, Article ID D23111, 2012.

[11] D. T. Kleist and K. Ide, "An OSSE-based evaluation of hybrid variational-ensemble data assimilation for the NCEP GFS. Part I: system description and 3D-hybrid results," Monthly Weather Review, vol. 143, no. 2, pp. 433-451, 2015.

[12] L. Garand, J. Feng, S. Heilliette, Y. Rochon, and A. P. Trishchenko, "Assimilation of circumpolar wind vectors derived from highly elliptical orbit imagery: impact assessment based on observing system simulation experiments," Journal of Applied Meteorology and Climatology, vol. 52, no. 8, pp. 18911908, 2013.

[13] H. Wang, X. Huang, and Y. Chen, "An observing system simulation experiment for the impact of MTG candidate infrared sounding mission on regional forecasts: system development and preliminary results," ISRN Meteorology, vol. 2013, Article ID 971501, 18 pages, 2013.

[14] S. Heilliette, Y. J. Rochon, L. Garand, and J. W. Kaminski, "Assimilation of infrared radiances in the context of observing system simulation experiments," Journal of Applied Meteorology and Climatology, vol. 52, no. 4, pp. 1031-1045, 2013.
[15] O. Reale, J. Terry, M. Masutani, E. Andersson, L. P. Riishojgaard, and J. C. Jusem, "Preliminary evaluation of the European Centre for Medium-Range Weather Forecasts (ECMWF) nature run over the tropical Atlantic and African monsoon region," Geophysical Research Letters, vol. 34, no. 22, Article ID L22810, 2007.

[16] M. Masutani, T. W. Schlatter, R. M. Errico et al., "Observing system simulation experiments," in Data Assimilation: Making Sense of Observations, W. Lahoz, B. Khattatov, and R. Menard, Eds., pp. 647-679, Springer, Berlin, Germany, 2010.

[17] M. Masutani, J. S. Woollen, S. J. Lord et al., "Observing system simulation experiments at the National Centers for Environmental Prediction," Journal of Geophysical Research, vol. 115, Article ID D07101, 2010.

[18] D. M. Barker, W. Huang, Y.-R. Guo, A. J. Bourgeois, and Q. N. Xiao, "A three-dimensional variational data assimilation system for MM5: implementation and initial results," Monthly Weather Review, vol. 132, no. 4, pp. 897-914, 2004.

[19] Y. Rochon, J. W. Kaminski, S. Heilliette, L. Garand, J. de Grandpré, and R. Ménard, "Observation system simulation experiment for the PREMIER mission," in Proceedings of the 5th WMO Workshop on the Impact of Various Observing Systems on Numerical Weather Prediction, Sedona, Ariz, USA, May 2012, http://www.wmo.int/pages/prog/www/OSY/Reports/NWP-5_ Sedona2012.html.

[20] L. Garand, M. S. Buehner, S. Heilliette, S. R. Macpherson, and A. Beaulne, "Satellite radiance assimilation impact in new Canadian ensemble-variational system," in Proceedings of the Eumetsat Meteorological Satellite Conference, Vienna, Austria, September 2013, http://www.emetsoc.org/.

[21] L. Garand, "Toward an integrated land-ocean surface skin temperature analysis from the variational assimilation of infrared radiances," Journal of Applied Meteorology, vol. 42, no. 5, pp. 570-583, 2003.

[22] C. Cardinali, J. Pailleux, and J.-N. Thépaut, "Use of simulated Doppler wind lidar data in NWP: an impact study," Note de Travail du Groupe de Modelisation pour l'Assimilation et le Prevision 6, Centre National de la Recherche Scientifique, Météo-France, 1998.

[23] M. Masutani, J. S. Woollen, S. J. Lord et al., "Observing system simulation experiments at the National Centers for Environmental Prediction," Journal of Geophysical Research D: Atmospheres, vol. 115, no. 7, Article ID D07101, 2010.

[24] A. Stoffelen, G. J. Marseille, F. Bouttier, D. Vasiljevic, S. de Haan, and C. Cardinali, "ADM-Aeolus Doppler wind lidar observing system simulation experiment," Quarterly Journal of the Royal Meteorological Society, vol. 132, no. 619, pp. 1927-1947, 2006.

[25] M. Buehner, R. A. MacTaggart-Cowan, C. Beaulne et al., "Implementation of deterministic weather forecasting systems based on ensemble-variational data assimilation at Environment Canada. Part I: the global system," Monthly Weather Review, vol. 143, 2015. 

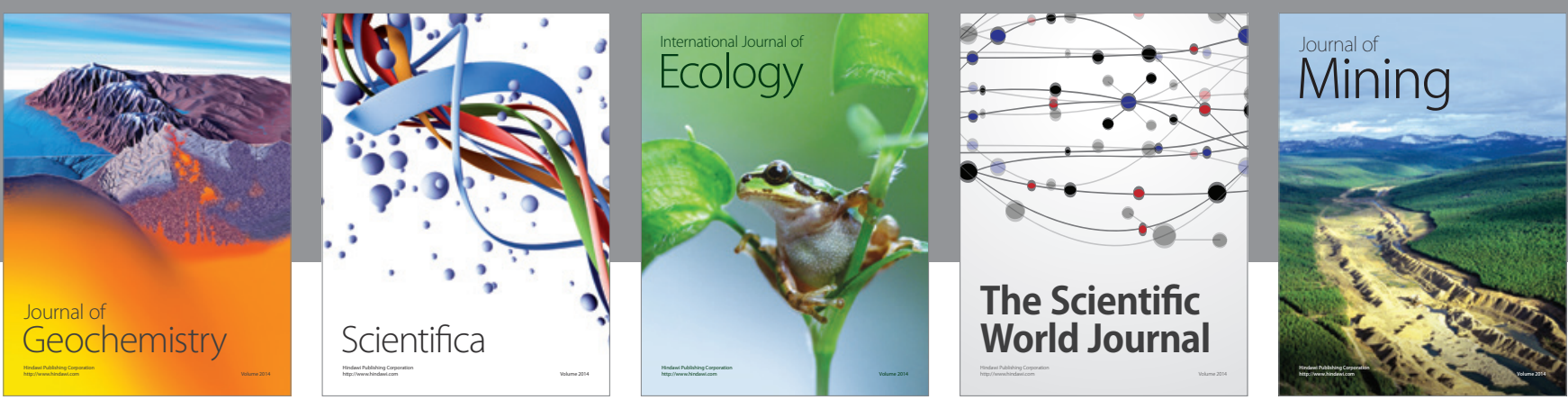

The Scientific World Journal
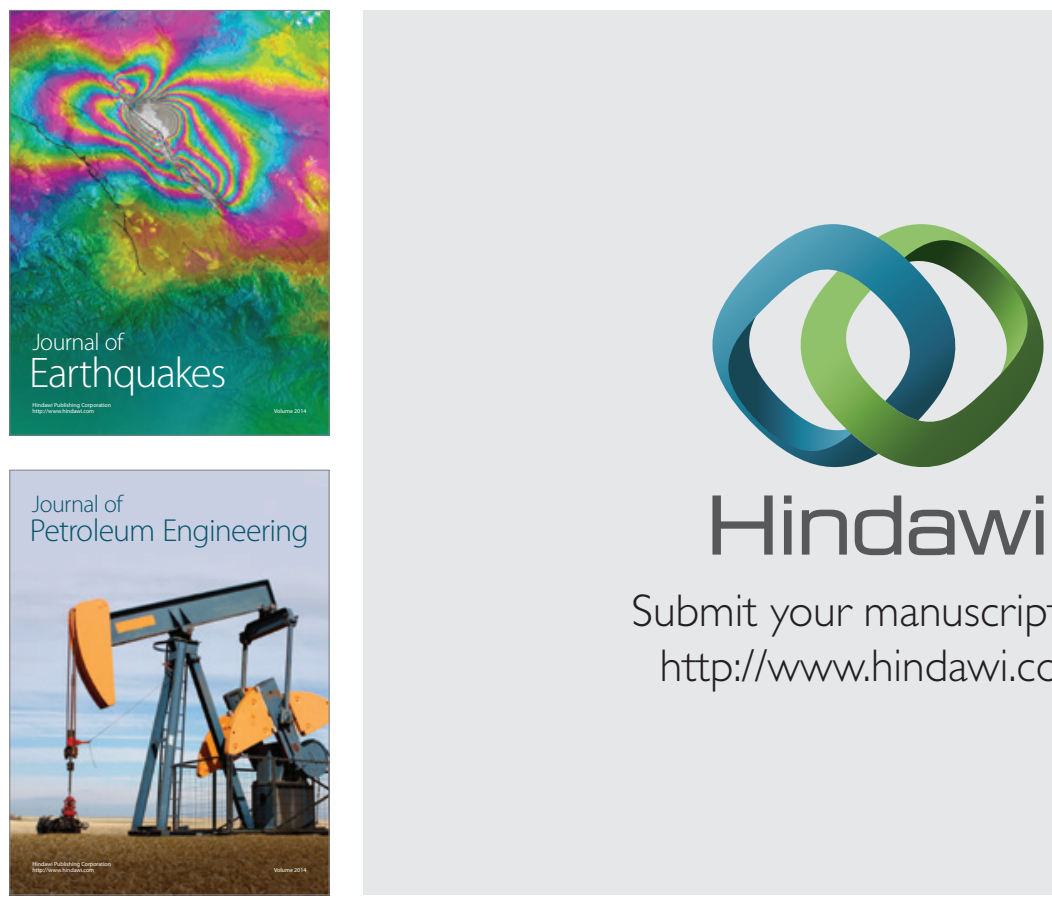

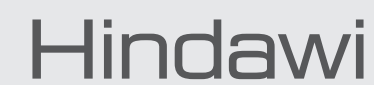

Submit your manuscripts at

http://www.hindawi.com
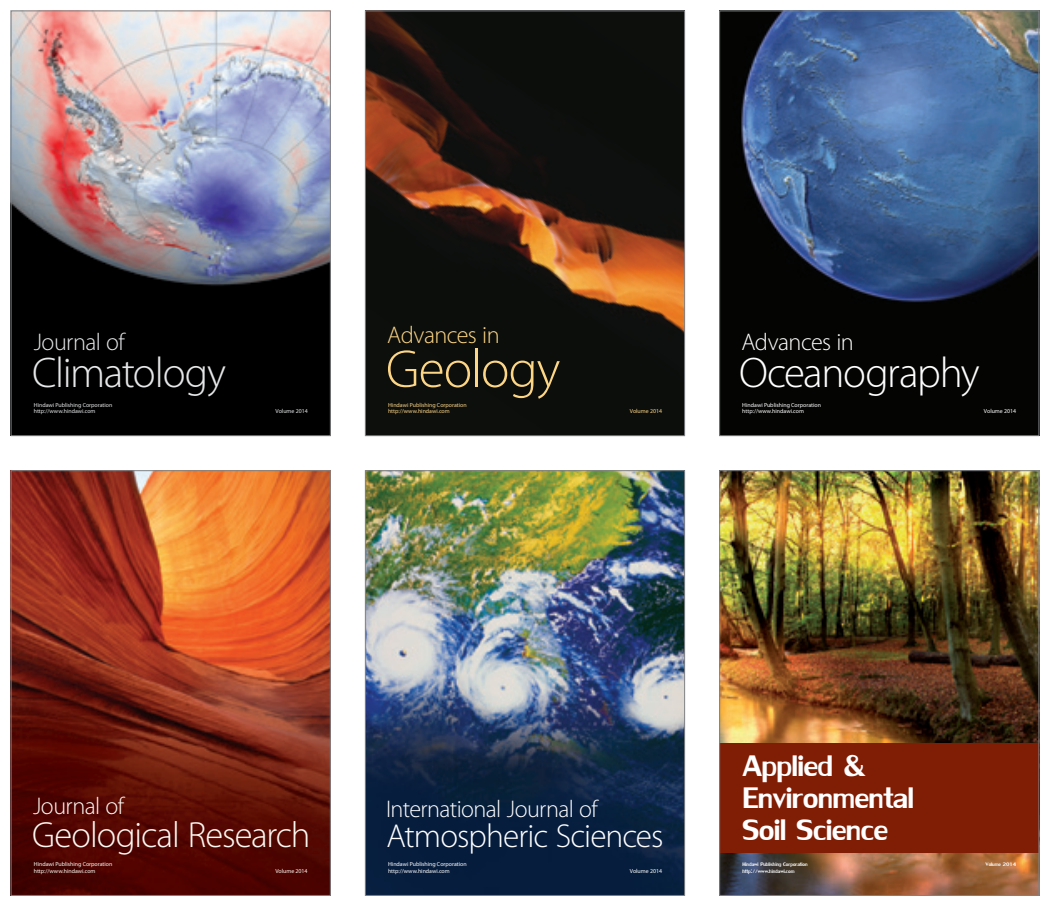
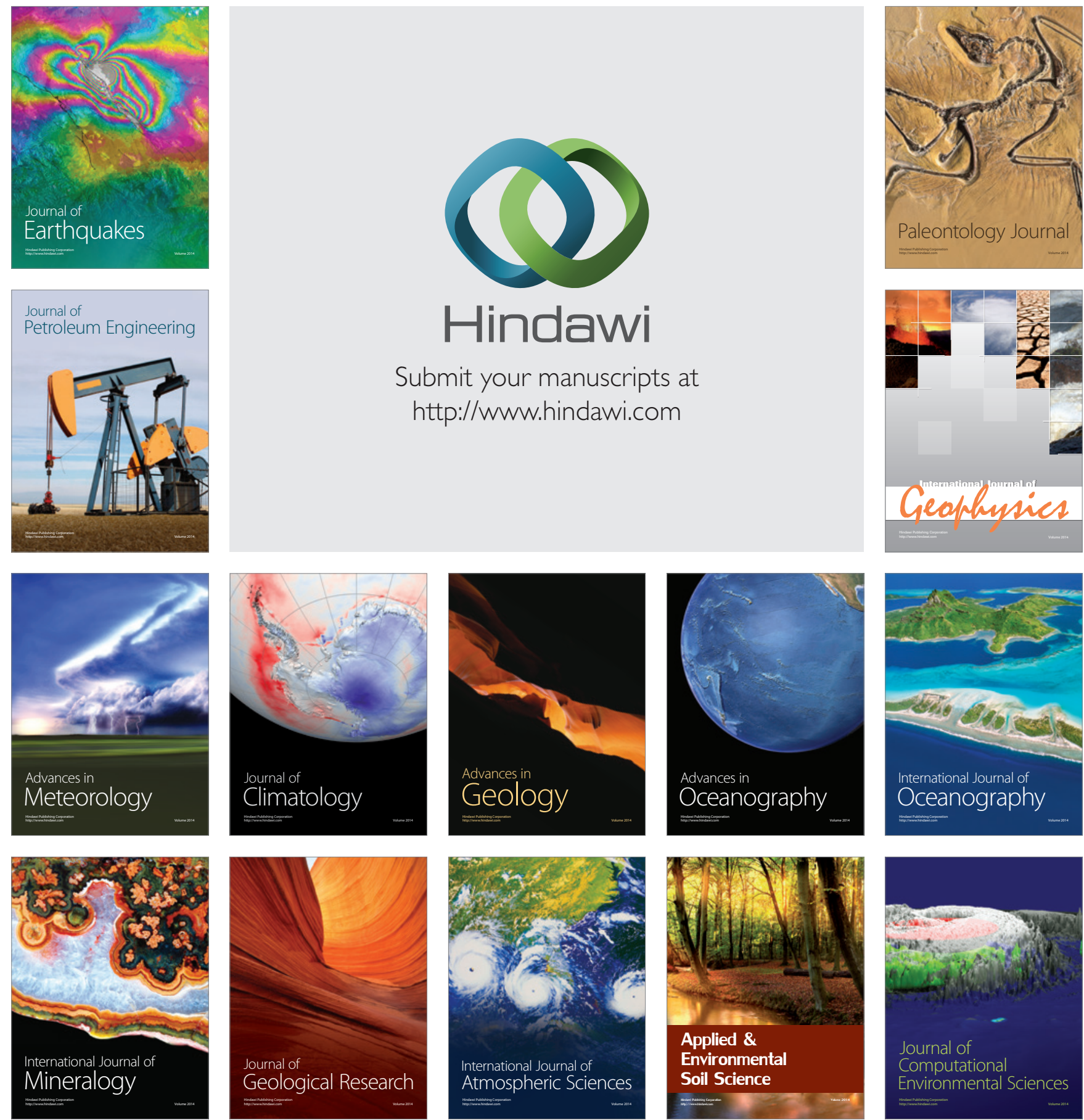